\title{
Towards developing product applications of thick origami using the offset panel technique
}

\author{
Michael R. Morgan ${ }^{1}$, Robert J. Lang ${ }^{2}$, Spencer P. Magleby ${ }^{1}$, and Larry L. Howell ${ }^{1}$ \\ ${ }^{1}$ Department of Mechanical Engineering, Brigham Young University, Provo, UT 84602, USA \\ ${ }^{2}$ Lang Origami, Alamo, CA 94507, USA \\ Correspondence to: Spencer P. Magleby (magleby@byu.edu)
}

Received: 15 September 2015 - Revised: 21 December 2015 - Accepted: 1 February 2016 - Published: 7 March 2016

\begin{abstract}
Several methods have been developed to accommodate for the use of thick materials in origami models which preserve either the model's full range of motion or its kinematics. The offset panel technique (OPT) preserves both the range of motion and the kinematics while allowing for a great deal of flexibility in design. This work explores new possibilities for origami-based product applications presented by the OPT. Examples are included to illustrate fundamental capabilities that can be realized with thick materials such as accommodation of various materials in a design and manipulation of panel geometry resulting in an increased stiffness and strength. These capabilities demonstrate the potential of techniques such as the OPT to further inspire origami-based solutions to engineering problems.
\end{abstract}

\section{Introduction}

In recent years, origami's potential for innovative solutions facilitated by its complex behaviors, yet simple fabrication methods has caught the attention of scientists and engineers. Some of the proposed applications of origami include deployable space applications (Schenk and Guest, 2011; Zirbel et al., 2013; Wu and You, 2010), nano-structure fabrication (Arora et al., 2006), robotics (Felton et al., 2014), and medical equipment (Kuribayashi et al., 2006; Francis et al., 2014). While origami's potential can be seen and even explored using traditional paper origami, many engineering applications would generally require materials with stiffness and strength. With increased stiffness and strength, however, comes some degree of thickness, and as thickness increases folding becomes less and less feasible.

Several formal methods have been developed for accommodating thickness in origami-based design. These include methods that shift rotational axes to edges of panels (Tachi, 2011; Hoberman, 2010), mount trimmed panels onto membranes (Zirbel et al., 2013), employ spatial mechanisms at vertices (Chen et al., 2015), taper the edges of the panels (Tachi, 2011), and replace creases with a rigid link and two axes to allow folding of adjacent panels (Ku and Demaine,
2015). The method used in this paper, the offset panel technique (OPT) developed by Edmondson et al. (2014), preserves the kinematics of an origami model and allows a full range of motion. While the benefits of these two traits can come at the cost of self-intersection and some complexity, the technique does allow for a variety of shapes, materials, and movements to be employed in a thick model based on a given origami pattern.

Previously, a number of basic configuration-related capabilities of the OPT have been shown by Edmondson et al. (2015). For example, the technique can provide designers with flexibility as it accommodates uniform and varying panel thickness, gaps between panels, and freedom in joint plane placement. In this paper, the intent of the authors is to move beyond describing the basic capabilities of the OPT (to accommodate thick panels) to illustrating how the technique can be used to facilitate the development and engineering of origami-based devices and products. The examples visually demonstrate that employing the OPT with various origami patterns can facilitate the design of mechanisms that use a variety of materials, have links (panels) that are non-planar shapes, and that can resist loads and/or transfer forces. Engineers and designers can use the illustrated examples as inspiration for further designs that use origami patterns. 
Table 1. A comparison of thickness accommodation methods.

\begin{tabular}{lccccc}
\hline Method & $\begin{array}{c}\text { Kinematics } \\
\text { preserved }\end{array}$ & $\begin{array}{c}\text { ROM } \\
\text { preserved }\end{array}$ & Single DOF & $\begin{array}{c}\text { Unfolds } \\
\text { flat }\end{array}$ & Application considerations \\
\hline $\begin{array}{l}\text { Axis-shift } \\
\text { Tachi (2011) }\end{array}$ & No & Yes & Yes & Yes & Limited to selected fold patterns \\
\hline $\begin{array}{l}\text { Offset joint } \\
\text { Hoberman (1991) } \\
\text { Hoberman (2010) }\end{array}$ & No & Yes & Yes & $\begin{array}{c}\text { Yes, } \\
\text { varied } \\
\text { thickness }\end{array}$ & Limited to selected fold patterns \\
$\begin{array}{l}\text { Membrane folds } \\
\text { Zirbel et al. (2013) }\end{array}$ & No & $\begin{array}{c}\text { Yes, if gaps } \\
\text { between panels } \\
\text { 2 thickness }\end{array}$ & $\begin{array}{c}\text { Now gaps } \\
\text { allowement }\end{array}$ & Yes & $\begin{array}{l}\text { Deployed system requires } \\
\text { tension at edges to keep } \\
\text { membranes stretched }\end{array}$ \\
\hline $\begin{array}{l}\text { Tapered panels } \\
\text { Tachi (2011) }\end{array}$ & Yes & No & Yes & Yes & $\begin{array}{l}\text { Required tapering of panels limits } \\
\text { possible geometry and materials }\end{array}$ \\
\hline $\begin{array}{l}\text { Offset crease } \\
\text { Abel et al. (2015) }\end{array}$ & No & Yes & No & Yes & $\begin{array}{l}\text { Panels required to be trimmed to } \\
\text { avoid self-interference at vertices }\end{array}$ \\
\hline $\begin{array}{l}\text { Spatial linkages } \\
\text { Chen et al. (2015) }\end{array}$ & No & Yes & Yes & No & $\begin{array}{l}\text { Fold angles and panel thicknesses } \\
\text { limited by the spatial mechanism }\end{array}$ \\
\hline $\begin{array}{l}\text { Offset panel } \\
\text { Edmondson et al. (2014) }\end{array}$ & Yes & Yes & Yes & No & $\begin{array}{l}\text { Cutouts in panels may be required } \\
\text { to avoid self-intersection }\end{array}$ \\
\hline
\end{tabular}

\section{Background}

As preparation for extending the application of the OPT, we briefly review three areas. These areas combined lead to an understanding of new ways to create mechanical products by adapting origami models and motions for thick materials.

\subsection{Modeling origami}

To take advantage of an origami mechanism's potential for application, it is useful to have mathematical models of its motion and behaviors. Origami can be modeled as a web of coupled spherical mechanisms where each vertex is the center of a spherical mechanism, each panel is a link, and each fold is a joint (e.g. Greenberg et al., 2011; Bowen et al., 2013; Lang and Hull, 2005; Evans et al., 2015; Abel et al., 2015; Tachi, 2009a; Miura, 1989). Spherical kinematic mechanisms belong to a subset of three-dimensional kinematics in which any point on the mechanism is constrained to be coincident with a spherical surface whose center is the point of intersection of all joints within the mechanism. The behavior of a spherical mechanism is defined by the location of the rotational axes. In other words, if a link's shape or size changes, as long as the rotational axes have not been altered, the motion will be the same (Chiang, 2000; Bowen et al., 2014).

\subsection{Thickness accommodation}

Mathematical models have been developed which have been used to predict the behavior of a given fold pattern (Tachi, $2009 \mathrm{~b}$ ) and which can generate crease patterns based on a desired form (Tachi, 2009a; Lang, 1996). These mathematical models usually assume zero-thickness materials. Due to paper's relatively thin nature, zero-thickness is a suitable approximation for traditional origami. However, this approximation breaks down for thicker origami materials (Francis et al., 2013).

Several techniques have been developed to accommodate thick materials. Each of the thickness accommodation techniques has its own set of strengths, weaknesses, and limitations. Figures 1 and 2 illustrate seven methods, including the OPT, and Table 1 lists the methods and compares them against four characteristics that will be important to discussing applications for the OPT. Following is a description of these characteristics.

Kinematics preserved indicates if the kinematics of the base origami model (a zero-thickness model) is preserved with the method. While matching kinematics may not be important for some applications, in many it will be essential to achieve the same degrees of freedom, the consistency, or the predictability of a motion identical to the origami model.

ROM preserved indicates whether or not the range of motion, from fully folded to fully deployed, is preserved. Many methods do not allow for full range of motion due to clashes of panels/edges. In some applications full 


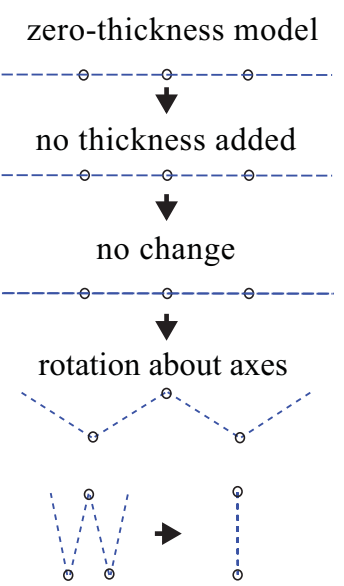

(a)

zero-thickness model

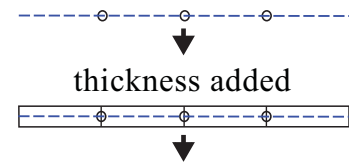

tapered to rotational axes

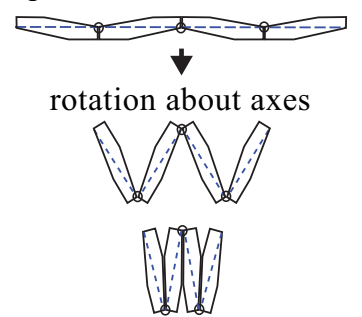

(d) zero-thickness model

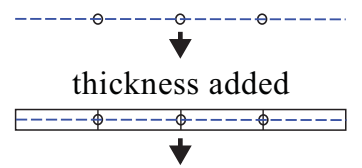

shift rotational axes to edge

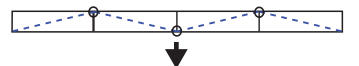

rotation about axes
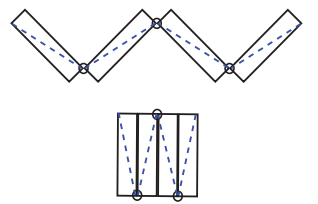

(b)

zero-thickness model

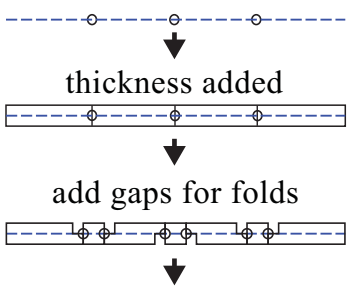

rotation about axes

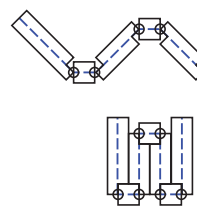

(e) zero-thickness model

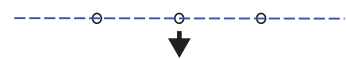

thickness added on membrane

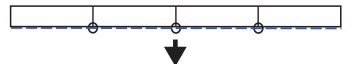

add gaps for valley folds

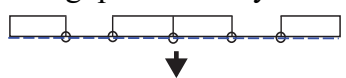

rotation about axes

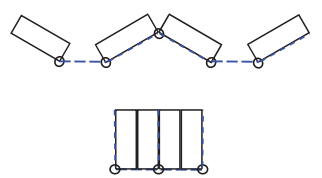

(c)

zero-thickness model

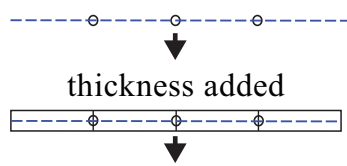

offset thick panels

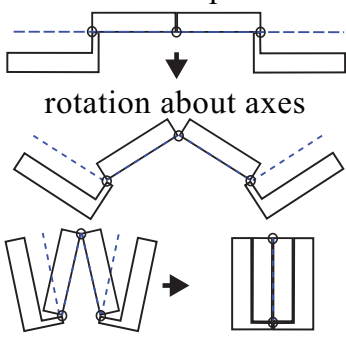

(f)

Figure 1. An illustration of the concepts of different thickness accommodation methods. All images, except (e), are from Edmondson et al. (2014). (a) The zero-thickness model describes the basic kinematic behavior of the model. (b) The axis-shift method as demonstrated by Tachi (2011) shifts each rotational axis to either the top or bottom of the thick material. While slightly different conceptually, the method described by Hoberman (2010) can be illustrated identically. (c) The membrane folds method by Zirbel et al. (2013) mounts thick-material facets to a flexible membrane. (d) The tapered panels method from Tachi (2011) trims material from the panel edges to maintain the kinematics. (e) The offset crease technique, described by Abel et al. (2015), is similar to the membrane folds method, but calls for rigid material in the gaps between panels. This method was inspired by work done by Hoberman (1991) (f) The offset panel technique shown by Edmondson et al. (2014) offsets each panel from a selected joint plane and extends the rotational axes back to the joint plane.

motion may not be required, but in many there is a need for the folds to move through the full $180^{\circ}$.

Single DOF indicates if the method will result in a single degree-of-freedom (DOF) system (assuming that the pattern itself has a single DOF). Many rigid-foldable origami models have one DOF. For many applications, especially those implementing a deployable application, a single DOF is desirable.

Unfolds flat indicates whether or not the thick origami system resulting from the method will be flat in its fully unfolded shape. Flat is defined in this case as resulting in a system where the entire upper face of each panel lies in the same plane. Traditional paper origami unfolds flat by this definition. Most of the methods do not produce fully flat configurations as the panels are offset or tapered. The OPT does not result in flat configurations, but the offset panels (or panel substitutes) can be parallel to the joint plane.

Application considerations presents notes on key aspects which should be considered when creating practical applications with the method. Depending on the application, the considerations may be impediments or used as advantages. 


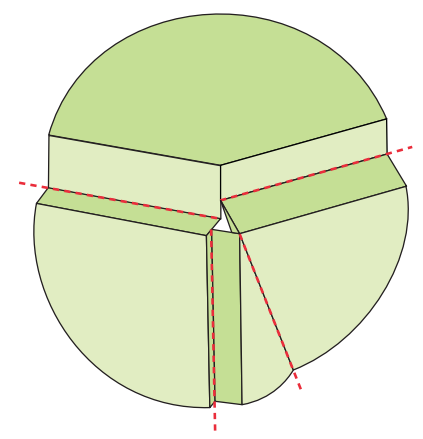

Figure 2. Chen et al. (2015) demonstrates a thickness accommodation method which uses spatial linkages at the vertices of the origami pattern to achieve its motion. A single origami vertex is shown that employs such a mechanism. This illustration is separate because the method cannot be portrayed in the same twodimensional form as the other methods. The red dotted lines indicate the rotational axes. This method was shown to work with degree-4, degree-5, and degree- 6 vertices.

In this paper, the applications that are presented take advantage of the OPT's capabilities to preserve kinematics, allow for full range of motion, and open with a single DOF. OPT-based systems generally do not fold flat and have a tiered appearance in the open position. This is used as an advantage in some of the following examples.

\subsection{Offset panel technique}

One of the major advantages of the OPT is that it maintains both the kinematic behavior and the full $180^{\circ}$ of motion as demonstrated by Edmondson et al. (2014). The former permits designers to take advantage of the mathematical models already developed while the latter allows for fully opened and closed models. The OPT also allows for flexibility in a design. Since an origami-inspired design is constrained by only the preservation of the location of the axes and selfintersection, attributes such as varied panel thickness, spacing between panels, and selectable joint plane placement are all possible with the OPT (see Edmondson et al., 2015).

\section{Fundamental capabilities of the offset panel technique}

A major benefit of traditional origami is the simplicity that it offers - the desired model is a product of a single monolithic piece of paper which undergoes no process other than folding. While some of this simplicity is lost in "thick origami", there are at least two fundamental capabilities possessed by origami models implemented by the OPT which are not exhibited by traditional origami. These are the variation of panel geometry and the material(s) used in a design. We refer to these as fundamental capabilities because others are either subsets of them or results of combining them.

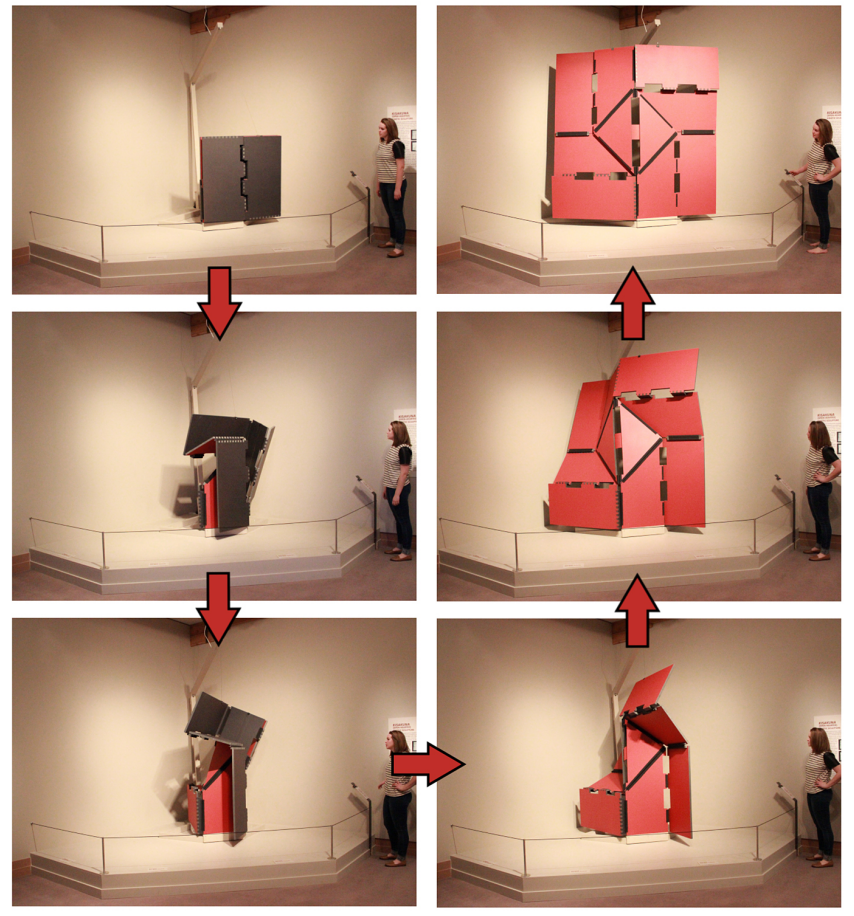

Figure 3. The unfolding of an origami-based kinetic sculpture which employs the OPT. The mechanism is made of panels with a thickness of one inch. This was part of an exhibit in BYU's Museum of Art.

\subsection{Panel and joint materials}

With the option of using non-paper materials, it becomes clear that virtually any solid material can be utilized in designs that incorporate folding. Designers of origami-inspired products now have the ability to accommodate materials that are, for example, transparent/opaque, conductive/insulative, lubricative/abrasive, adhesive, stiff, modifiable, expansive, electrically charged, absorbent, or reflective.

Additionally, not only can a model be made of virtually any solid material, since thick origami panels are fabricated individually in most cases, the individual panels of a model can be assigned different materials. For example, Fig. 3 shows a kinetic sculpture based on an origami fold pattern. In the design of this model, a lightweight material was desired for the moving panels, while strength was needed in the supporting ground panel. A lightweight foam board was chosen to be used for the moving panels and MDF was used for the ground panel. The different sculpture materials can best be seen from the back, as shown in Fig. 4.

This kinetic sculpture is based on the square twist fold pattern shown in Fig. 5a. Figure 5b illustrates the closed stack of panels (looking down at the sculpture from the top). In this design, the panels all have an equal thickness of one inch and, while interference has been accounted for, the panel profiles have not been altered. Figure 5c shows the same panel stack but includes the offsets which have the same color as the pan- 


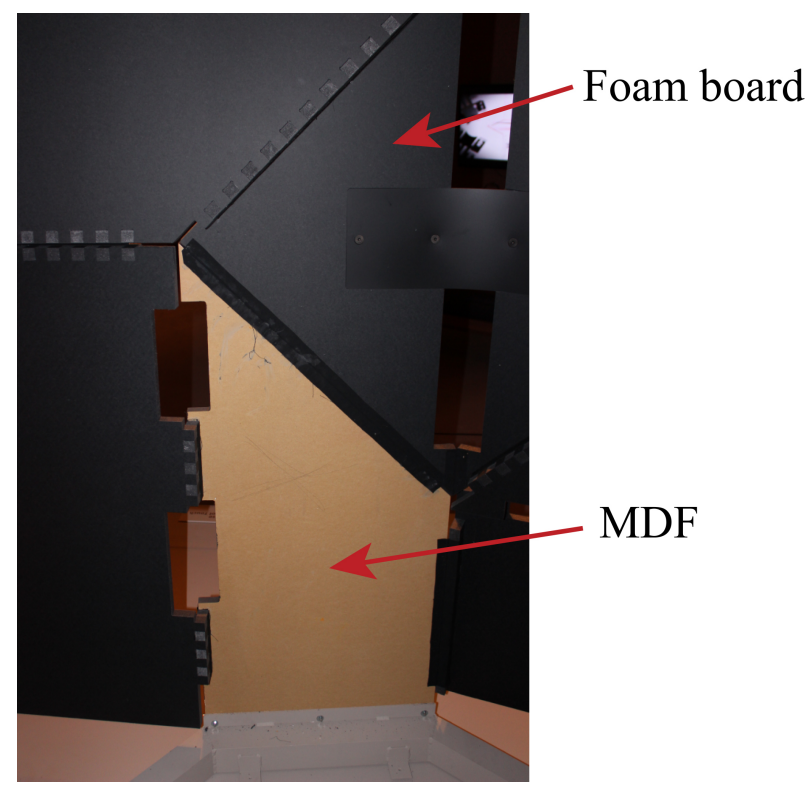

Figure 4. In the kinetic sculpture, the grounded panel is made of MDF while all others are made of a foam board.

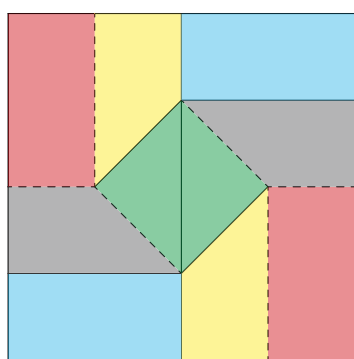

(a)

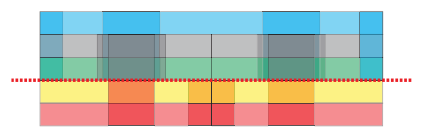

(c)

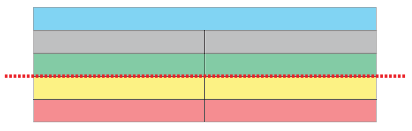

(b)

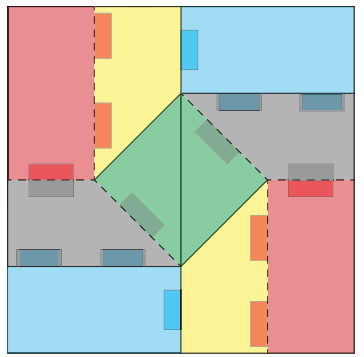

(d)
Figure 5. A schematic of the pattern used in the design of the kinetic sculpture. (a) The square twist crease pattern. (b) A side view of the stacked panels and joint plane (represented by the red line). (c) The panel stack with offsets. (d) The unfolded panels including the offsets for the OPT model.

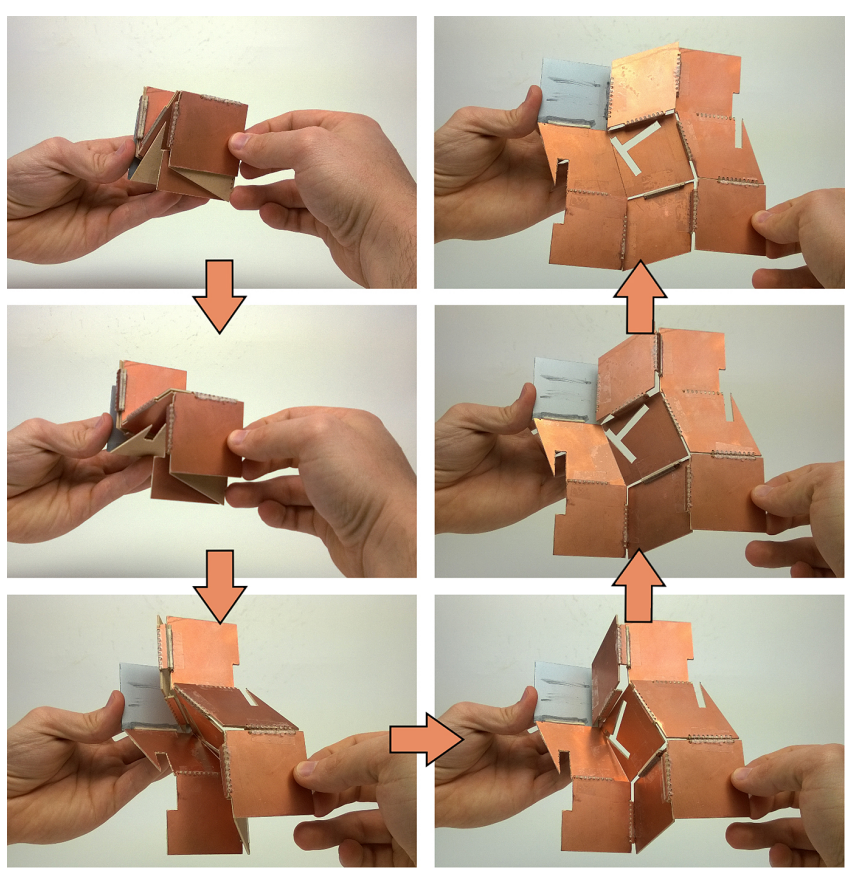

Figure 6. A foldable circuit board with one panel constructed from metal. The thickness of the panel is approximately 0.063 inches.

els to which they are rigidly attached. The offsets are shown to demonstrate the preservation of the axes' locations which in the closed configuration all lie on a single plane (referred to as the joint plane) indicated by the dotted red line.

The next example shows the possibilty of a foldable circuit board which uses the OPT. This is shown in Fig. 6. All but one panel is made from a PCB substrate, while the exception is made of a metal plate intended to act as the ground layer. While simpler crease patterns (i.e., a tesselated tri-fold pattern) could offer the similar stowed-to-deployed area ratios, this pattern facilitates connections between each panel and its neighbors and a single DOF.

A square twist pattern, as illustrated in Fig. 7a, was used as a base for this circuit board. The illustration in Fig. 7b shows the stacked panels from the side and Fig. 7c shows the same panel stack with the offsets.

\subsection{Panel geometry}

One of the most applicable and inspiring capabilities of thick origami (the OPT in particular) is the freedom a designer has to alter the geometry of the panels. Once it is determined that an origami-inspired design will employ the OPT, the only limitations are the preservation of the rotational axes and the prevention of self-intersection. Panel material can be added and/or removed to add structure, give form, achieve motion, etc. Combining this idea with that of using various materials, not only is it possible to alter the shape of a model's panel material, but panels can be replaced entirely by mechanical parts, electrical parts, displays, wheels, optical devices, solar 


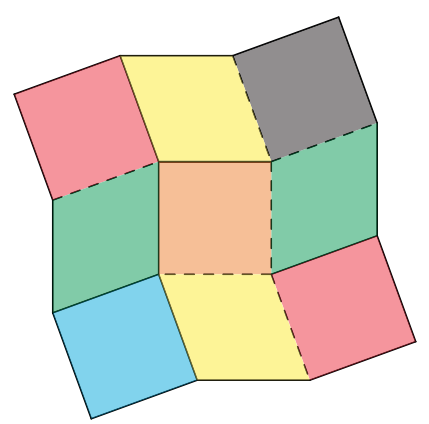

(a)
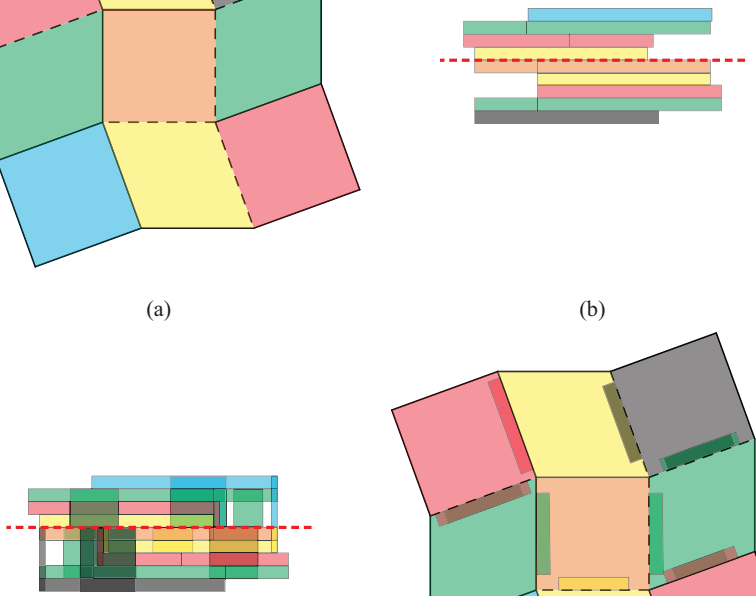

(c)

(d)

Figure 7. A schematic of (a) the pattern used in the design of the foldable circuit board. (b) A side view of the panel stack and the joint plane. (c) The stacked panels with offsets. (d) The unfolded pattern with the offsets. (b) and (c) are $2 \mathrm{X}$ scale.

panels, molds, etc. As long as axes are not moved and selfintersection is avoided, the model will maintain the kinematic behavior and the range of motion of the source model.

To demonstrate the manipulation of panel geometry, a model of a folding sphere is shown in Fig. 8. From the closed configuration of a basic OPT model with uniform thickness, material is added to each panel in such a way that the model takes on the shape of a sphere. This is done without affecting the rotational axes and with care taken to ensure no selfintersection.

A version of the square twist as shown in Fig. 9a formed the origami base of this foldable sphere. Figure $9 \mathrm{~b}$ and $9 \mathrm{c}$ show the panel stack alone and with the offsets, respectively, and Fig. 9d shows the panel stack with offsets and the material added to form a sphere.

As another example of non-constant panel geometry, see the engineer's toolbox in Fig. 10. This model explores the utility of adding and removing material by carving out material from the panels to form compartments for supplies and adding material to panels to form the walls of the box. The box's opening motion is shown in Fig. 11.

The same square twist pattern as used with the kinetic sculpture was used for the toolbox, but with a different crease assignment as shown in Fig. 12a. To facilitate the closing of the box, a small space was added between the panels as demonstrated by Edmondson et al. (2015). This spacing is

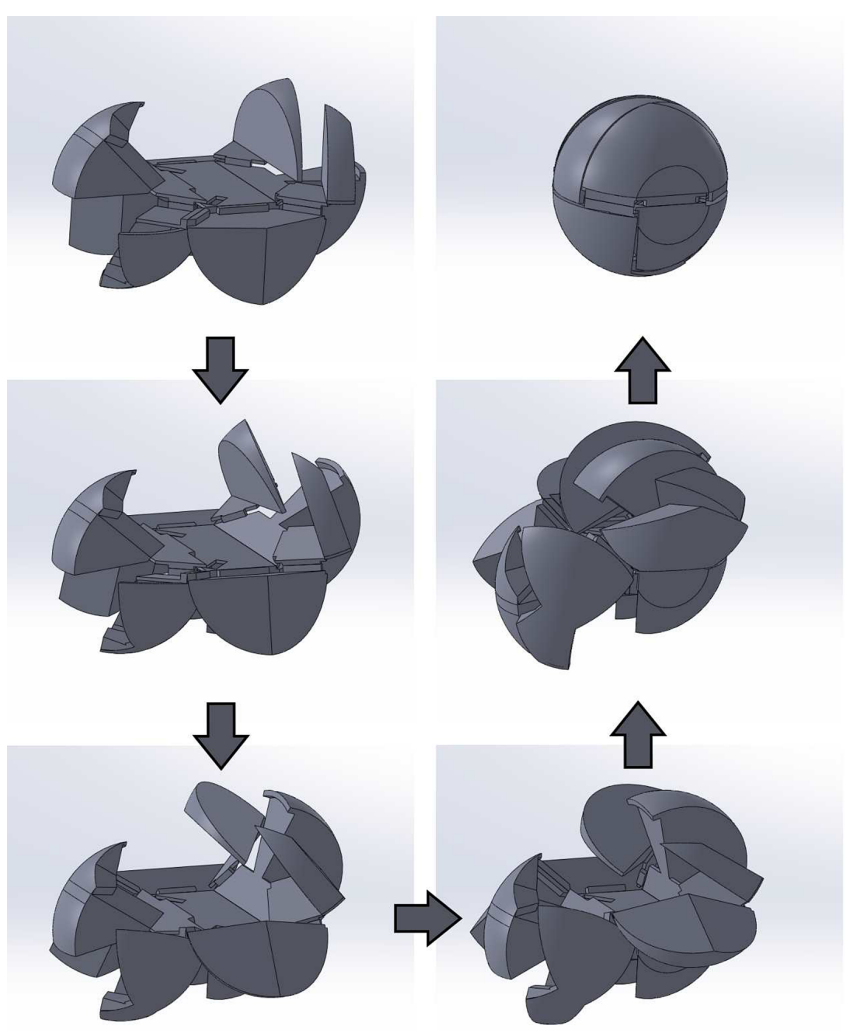

Figure 8. A kinematic simulation model of how panel geometry can vary, in this case to form a sphere.

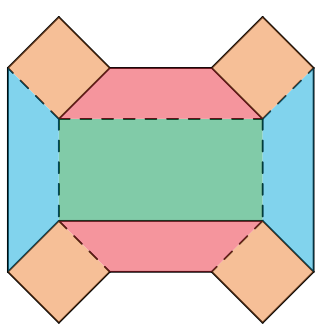

(a)

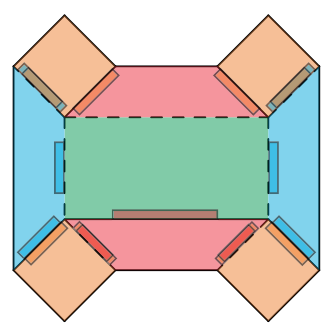

(d)

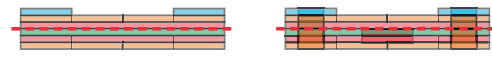

(b)

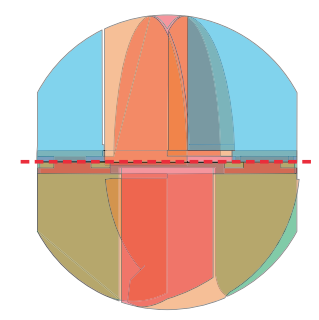

(e)
Figure 9. A schematic of the pattern used in the design of the foldable sphere. (a) The crease pattern. (b) The panel stack and joint plane. (c) The panel stack with offsets. (d) The offsets shown with the open model. (e) The panel stack with offsets and modified panel geometry. 


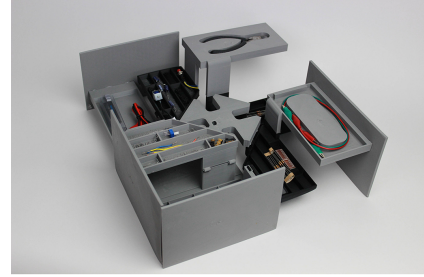

(a)

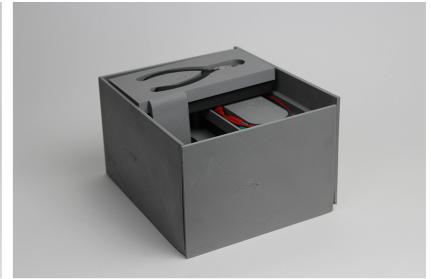

(b)
Figure 10. An electrical engineer's toolbox created from an origami crease pattern and the OPT in (a) the unfolded postion and (b) the folded position. The panel thickness in this model is 0.5 inches.

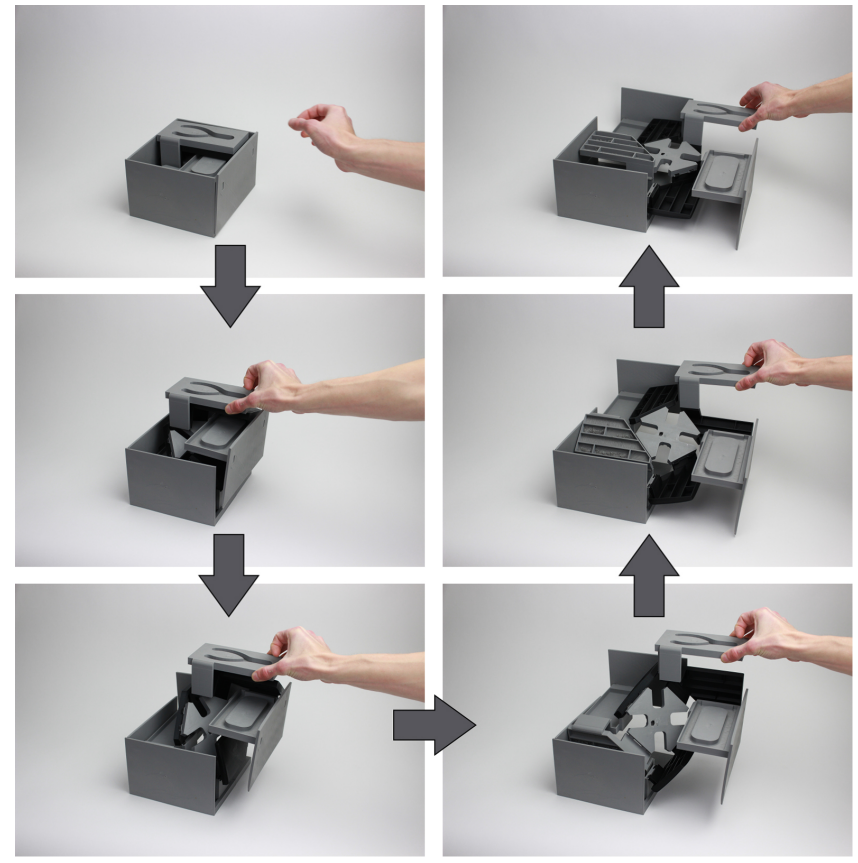

Figure 11. A demonstration of the motion of the toolbox.

shown in Fig. $12 \mathrm{~b}$ and the same panel stack is shown in Fig. 12c, but with offsets.

\section{Resultant capabilities - stiffness and strength}

Having discussed the use of various materials and geometry, it is now befitting to examine an example of a capability that is a result of simultaneously varying materials and geometry. We will explore the use of materials commonly used in engineering combined with the necessary geometry to develop designs which can support loads and apply forces. Based on the design specifications of an origami-inspired product, a fold pattern can be selected which would give the desired behavior - a particular motion, mechanical advantage, shape, or unfolded/folded size ratio. After the desired pattern has been determined, materials with sufficient stiffness and strength to

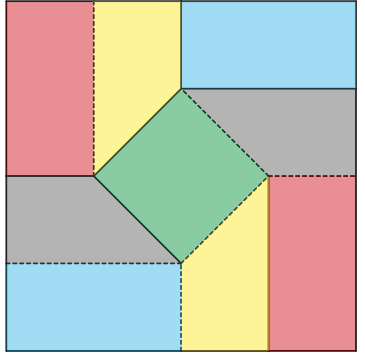

(a)

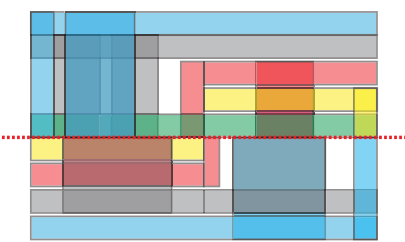

(c)

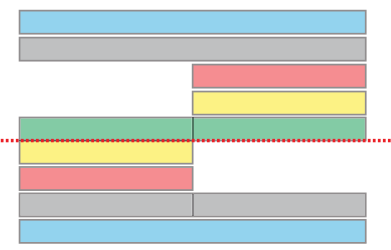

(b)

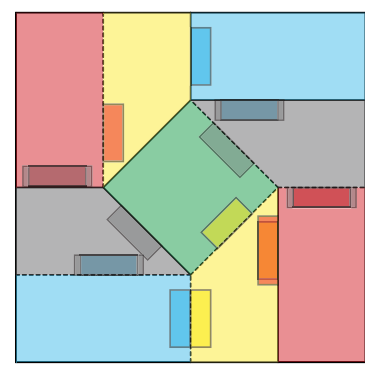

(d)
Figure 12. A schematic of the pattern used in the design of the toolbox. (a) The crease pattern. (b) The stacked panels and the joint plane (in red). (c) The stacked panels with added offsets. (d) The unfolded pattern including the offsets.

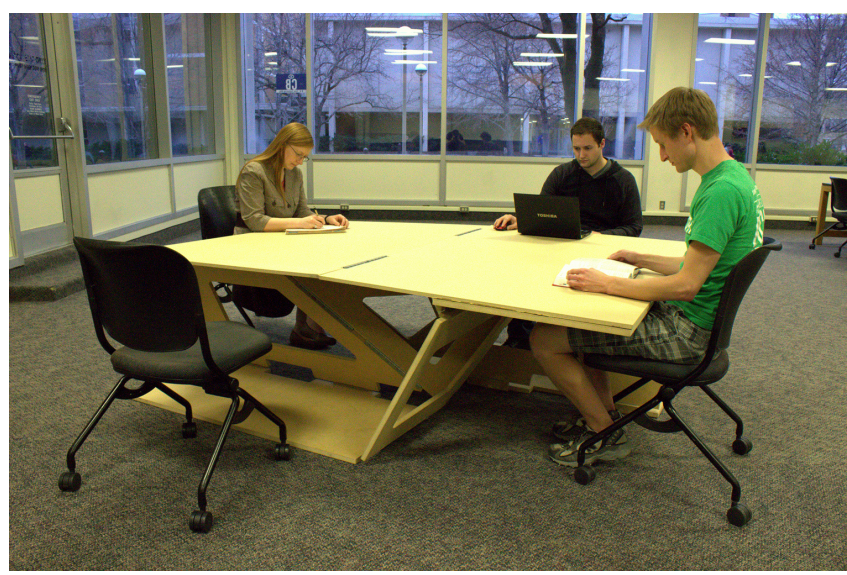

Figure 13. This origami-based table supports a significant amount of weight. Unlike the other examples in this paper, the table is designed to unfold to an intermediate position that does not correspond to the zero-thickness model's fully unfolded position. The panels in this design are 0.75 inches.

perform the desired task can be selected and any geometrical modifications necessary can be made.

For example, an origami-inspired table is shown in Fig. 13. In the folded configuration, the table is compact. In the unfolded configuration, the table is much larger and, based on the kinematics and the materials of the model, can support a significant amount of weight. The table's unfolding motion 


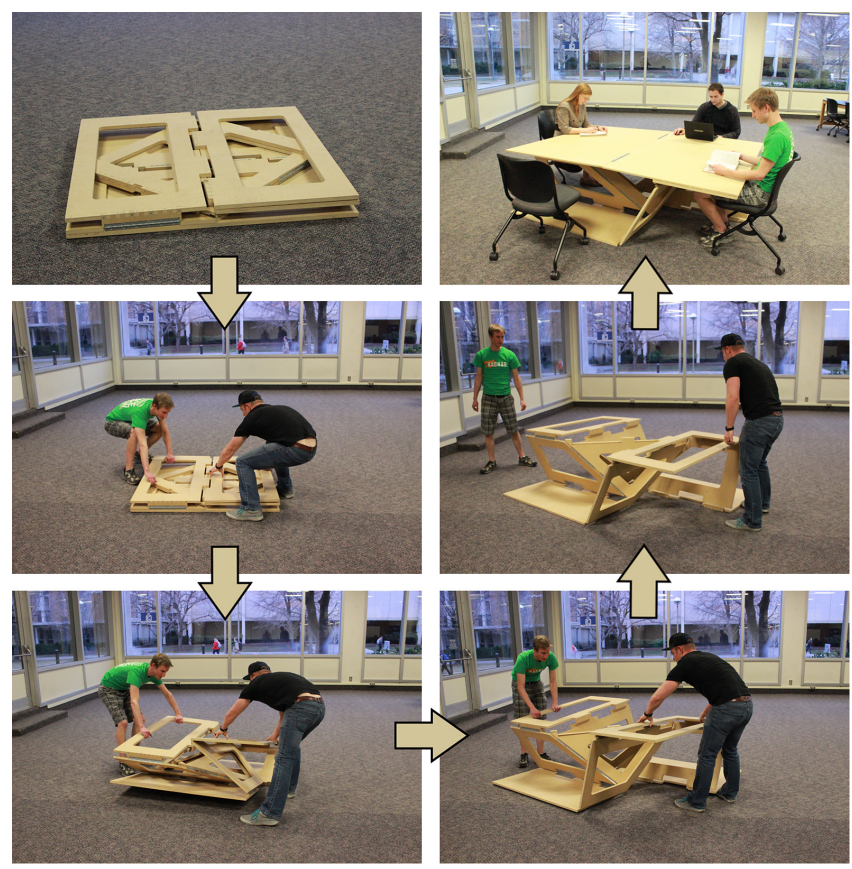

Figure 14. An origami-inspired table is shown through its opening motion.

is demonstrated in Fig. 14. The table uses the same square twist pattern as the kinetic sculpture and also has the same panel stacking configuration (see Fig. 5).

In the next example, we consider a lift. By using a single reverse fold, a substantial mechanical advantage is possible. In this case, the mechanical advantage is approximately 20 at the open state (top-left in Fig. 15) and gradually decreases to 5 at its highest point (top-right in Fig. 15). To withstand the stresses that accompany the loads and large mechanical advantage of this example, MDF was the selected material.

The lift employs a reverse fold which is a single vertex origami pattern as shown in Fig. 16a. Figure 16b shows the stack of the panels and Fig. 16c shows that same stack with the offsets and altered geometry. To create the offsets for this model, the entire inner faces of the long green panels were extended inward to the joint plane while leaving clearance for the small blue panels. This is equivalent to assigning different thicknesses to the panels as shown possible by Edmondson et al. (2015).

\section{Conclusions}

Origami's simple fabrication methods, infinite possibilities, and predictability provide it potential to emerge as a source of inspiration for many innovative designs. While paper origami models are useful for quickly visualizing and prototyping origami-inspired products, paper is often insufficient as a material for a finished product. However, the use of other materials in such origami-inspired designs often presents a

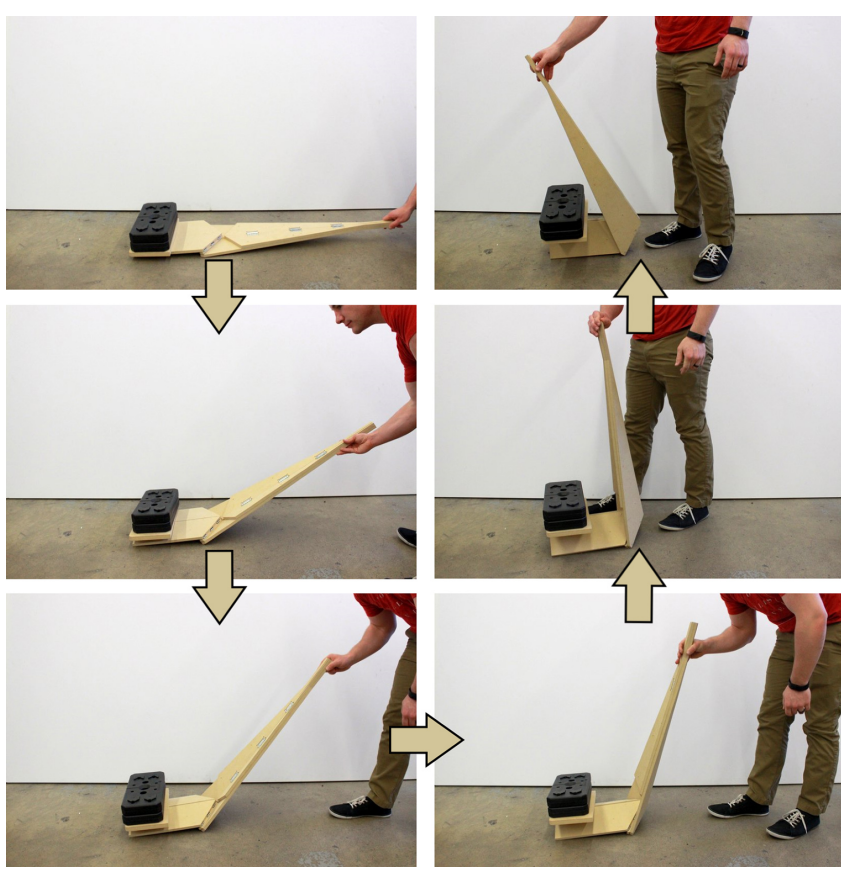

Figure 15. The origami-inspired lift mechanism shown through its motion as it lifts the black weight. The panels of the lift are 0.75 inches.

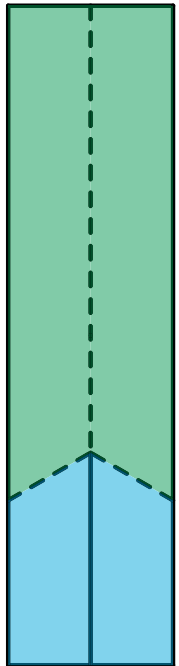

(a)

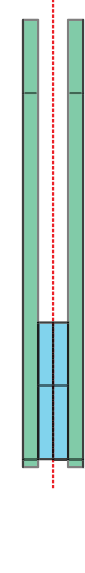

(b)

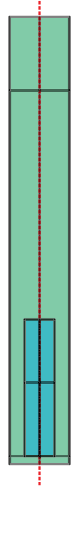

(c)

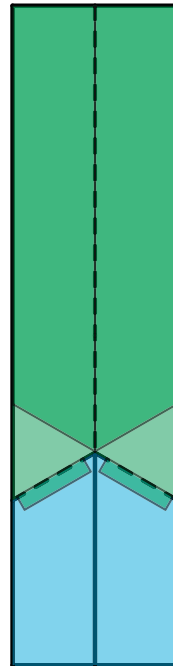

(d)
Figure 16. The schematic of the reverse fold pattern used in the design of the lift. The dotted red line represents the joint plane. (a) The fold pattern. (b) A side view of the panel stack. (c) The panel stack with offsets which in this design are large and cover the majority of the inside face of each green panel. (d) The open pattern with the offsets.

handful of major difficulties including the folding of these materials and the interference issues that accompany it. This paper has illustrated how designs based on origami patterns 
can be realized using non-paper materials in a variety of shapes and configurations. The examples show that common engineering-related product objectives can be achieved with origami-based mechanisms. The techniques shown in the examples can be applied to a wide variety of origami patterns.

Several methods for accommodating thickness have been developed, each with its own capabilities and limitations. While the examples in this paper use the offset panel technique, the capabilities of accommodation of various materials, manipulation of panel geometry, and strength and stiffness can be applied to other thickness accommodation methods. These ideas add new and exciting possibilities for origami-inspired design and facilitate the development of creative solutions to real-world problems.

Acknowledgement. This material is based on work supported by the National Science Foundation and Air Force Office of Scientific Research under NSF Grant EFRI-ODISSEI-1240417.

Edited by: G. Hao

Reviewed by: two anonymous referees

\section{References}

Abel, Z., Cantarella, J., Demaine, E. D., Eppstein, D., Hull, T. C., $\mathrm{Ku}$, J. S., Lang, R. J., and Tachi, T.: Rigid Origami Vertices: Conditions and Forcing Sets, arXiv.org, math.MG, available at: http://arxiv.org/abs/1507.01644v1 (last access: 22 July 2015), 2015.

Arora, W. J., In, H. J., Buchner, T., Yang, S., Smith, H. I., and Barbastathis, G.: Nanostructured Origami ${ }^{\mathrm{TM}}$ 3D Fabrication and Self Assembly Process for Soldier Combat Systems, Sel. Top. Electr. Syst., 42, 473-477, 2006.

Bowen, L. A., Grames, C. L., Magleby, S. P., Lang, R. J., and Howell, L. L.: A Classification of Action Origami as Systems of Spherical Mechanisms, J. Mech. Design, 135, 111008, doi:10.1115/1.4025379, 2013.

Bowen, L. A., Baxter, W., Magleby, S. P., and Howell, L. L.: A Position Analysis of Coupled Spherical Mechanisms Found in Action Origami, Mech. Mach. Theory, 77, 13-44, 2014.

Chen, Y., Peng, R., and You, Z.: Origami of thick panels, Science, 349, 396-400, doi:10.1126/science.aab2870, 2015.

Chiang, C. H.: Kinematics of Spherical Mechanisms, Krieger Publishing Company, Malabar, FL, 2000.

Edmondson, B. J., Lang, R. J., Magleby, S. P., and Howell, L. L.: An Offset Panel Technique for Thick Rigidly Foldable Origami, Proceedings of the ASME International Design Engineering Technical Conferences, Buffalo, NY, 18-20 August 2014, DETC201435606, 2014.

Edmondson, B. J., Lang, R. J., Morgan, M. R., Magleby, S. P., and Howell, L. L.: Thick Rigidly Foldable Structures Realized by an Offset Panel Technique, in: Origami 6, American Mathematical Society, 1, 149-161, 2015.

Evans, T. A., Lang, R. J., Magleby, S. P., and Howell, L. L.: Rigidly Foldable Origami Twists, in: Origami 6, AMS, 1, 119-130, 2015.
Felton, S., Tolley, M., Demaine, E., Rus, D., and Wood, R.: A method for building self-folding machines, Science, 345, 644646, doi:10.1126/science.1252610, 2014.

Francis, K. C., Blanch, J. E., Magleby, S. P., and Howell, L. L.: Origami-like creases in sheet materials for compliant mechanism design, Mech. Sci., 4, 371-380, doi:10.5194/ms-4-3712013, 2013.

Francis, K. C., Rupert, L. T., Lang, R. J., Morgan, D. C., Magleby, S. P., and Howell, L. L.: From crease pattern to product: Considerations to engineering origami-adapted designs, in: Proceedings of ASME 2014 International Design Engineering Technical Conferences \& Computers and Information in Engineering Conference, 17-20 August 2014, Buffalo, NY, 2014.

Greenberg, H. C., Gong, M. L., Magleby, S. P., and Howell, L. L.: Identifying links between origami and compliant mechanisms, Mech. Sci., 2, 217-225, doi:10.5194/ms-2-217-2011, 2011.

Hoberman, C.: Reversibly expandable structures, US Patent 4,981,732, United States Patent and Trademark Office, 1991.

Hoberman, C.: Folding Structures Made of Thick Hinged Panels, US 7794019, United States Patent and Trademark Office, 2010.

Ku, J. S. and Demaine, E. D.: Folding Flat Crease Patterns With Thick Materials, Proceedings of the ASME International Design Engineering Technical Conferences, 2-5 August 2015, Boston, MA, DETC2015-48039, 2015.

Kuribayashi, K., Tsuchiya, K., You, Z., Tomus, D., Umemoto, M., Ito, K., and Sasaki, M.: Self-deployable origami stent grafts as a biomedical application of Ni-rich TiNi shape memory alloy foil, Mat. Sci. Eng. A-Struct., 419, 131-137, 2006.

Lang, R.: A computational algorithm for origami design, in: Proceedings of the twelfth annual symposium on Computational geometry, ACM, 98-105, 1996.

Lang, R. J. and Hull, T. C.: Origami design secrets: mathematical methods for an ancient art, The Mathematical Intelligencer, 27, 92-95, 2005.

Miura, K.: A note on intrinsic geometry of origami, in: Proceedings of the First International Meeting of Origami Science and Technology, Ferrara, Italy, edited by: Huzita, H., 239-249, 1989.

Schenk, M. and Guest, S.: Origami Folding: A Structural Engineering Approach, in: Origami 5: Fifth International Meeting of Origami Science, Mathematics, and Education, edited by: WangIverson, P., Lang, R., and Yim, M., CRC Press, 291-304, 2011.

Tachi, T.: Origamizing Polyhedral Surfaces, IEEE T. Vis. Comput. Gr., 16, 298-311, doi:10.1109/tvcg.2009.67, 2009a.

Tachi, T.: Simulation of Rigid Origami, in: Origami 4: Fourth International Meeting of Origami Science, Mathematics, and Education, edited by Lang, R., A K Peters, Ltd., 175-188, 2009 b.

Tachi, T.: Rigid-Foldable Thick Origami, in: Origami 5: Fifth International Meeting of Origami Science, Mathematics, and Education, edited by: Wang-Iverson, P., Lang, R., and Yim, M., CRC Press, 253-264, 2011.

$\mathrm{Wu}, \mathrm{W}$. and You, Z.: Modelling rigid origami with quaternions and dual quaternions, P. Roy. Soc. A-Math. Phy., 466, 2155-2174, 2010.

Zirbel, S. A., Lang, R. J., Thomson, M. W., Sigel, D. A., Walkemeyer, P. E., Trease, B. P., Magleby, S. P., and Howell, L. L.: Accommodating Thickness in Origami-Based Deployable Arrays 1, J. Mech. Design, 135, 111005, doi:10.1115/1.4025372, 2013. 